\title{
Inteligencia emocional para mejorar el pensamiento crítico
}

\author{
Mg. Johanna del Carmen Romero Jiménez \\ P7001254326@ucvvirtual.edu.pe \\ ORCID: 0000-0001-8733-8191 \\ Universidad César Vallejo \\ Piura- Perú
}

Mg. Nancy Aracely Castro Ramírez P7001249979@ucvvirtual.edu.pe

ORCID: 0000-0003-3157-6320

Universidad César Vallejo

Piura- Perú
Mg. Wendy Yesenia Alvarado Torres
P7001249969@ucvvirtual.edu.pe
ORCID: 0000-0002-8133-8577
Universidad César Vallejo
Piura- Perú

Mg. Gina Marjorie Romero Jiménez

marjorie3062@hotmail.com

Guayaquil- Ecuador

\section{Mg. David Armando Mondragón Sánchez}

damondragonm@ucvvirtual.edu.pe

ORCID: 0000-0002-2025-3377

Universidad César Vallejo

Piura- Perú

\section{RESUMEN}

La investigación tuvo como objetivo plantear un programa de inteligencia emocional para mejorar el pensamiento crítico de los estudiantes de segundo año de bachillerato en ciencias de la Unidad Educativa Aida León Rodríguez Lara, Guayaquil 2021. La metodología de investigación fue de tipo aplicado, con un enfoque cuantitativo; y diseño no experimental de alcance transaccional, la muestra fue censal de 60 estudiantes, la técnica empleada para la recolección de datos fue la encuesta y como instrumento se utilizó un cuestionario para las dos variables de investigación, los instrumentos fueron validados según juicio de expertos y luego se procedió con la prueba de confiabilidad del Alfa Cronbach resultando para la variable independiente un nivel de 0,902 y para la variable dependiente 0,896 concluyéndose que los instrumentos son confiables. La conclusión más significativa es que los estudiantes exponen sus pensamientos tal cual los 
conciben sin detenerse a pensar acerca de las consecuencias de sus declaraciones ni a quienes pueden vulnerar originando problemas en la interrelación con sus compañeros, no suelen contribuir con soluciones frente a un problema debido a la falta de iniciativa, $\mathrm{y}$, por último, no saben influir sobre las personas para lograr salir de cualquier circunstancia logrando cosas a favor.

Palabras clave: inteligencia emocional; pensamiento crítico; clima escolar. 


\title{
Emotional intelligence to improve critical thinking
}

\begin{abstract}
The objective of the research was to propose an emotional intelligence program to improve the critical thinking of second year high school science students of the Aida León de Rodríguez Lara Educational Unit, Guayaquil 2021. The research methodology was of applied type, with a quantitative approach; and non-experimental design of transactional scope, the sample was a census of 60 students, the technique employee for data collection was the survey and as an instrument a questionnaire was used for the two research variables, the instruments were validated according to expert judgment and then proceeded with the reliability test of Cronbach's Alpha resulting for the independent variable a level of 0.092 and for the dependent variable 0.896 concluding that the instruments are truthful. The most significant conclusion is that students expose their thoughts as they conceive them without stopping to think about the consequences of their statements or those who may be harmed, causing problems in the interrelationship with the peers, they do not usually contribute with solutions to a problem due to lack of initiative. Finally, they do not know how to influence people to get out of any circumstance achieving things a favor.
\end{abstract}

Keywords: emotional intelligence; critical thinking; school climate.

Artículo recibido: 18. Julio. 2021

Aceptado para publicación: 08. Agosto. 2021 Correspondencia: P7001254326@ucvvirtual.edu.pe Conflictos de Interés: Ninguna que declarar 


\section{INTRODUCCIÓN}

La inteligencia emocional es una edificación que ayuda a comprender los diferentes cambios emocionales del entorno que nos rodea. La Organización de las Naciones Unidas para la Educación, la Ciencia y la Cultura, ([UNESCO], 2017) señala que la inteligencia emocional nos permite reconocer las emociones propias, soportar diversas tensiones y adversidades que ocurren día a día, aumentar la cooperación en equipo y tomar una postura empática y social que se incrementará el desarrollo personal. Fernández \& Montero (2015) enfatiza que las emociones e inteligencia han podido observarse con atención como discordantes o contradictorias, debido a que se pensaba que las emociones lograban perturbar a la inteligencia; Miners \& Hideg (2015) manifiesta que la inteligencia emocional es una breve historia pero en desorden; si bien ha tenido altos niveles de debates científicos, a pesar de esto ha sido centro de ataques por parte de sus críticos. Para Akbarilakeh et al (2018) la inteligencia emocional y el pensamiento crítico son principios para lograr la superación personal. De acuerdo con Trotta (2018) el 90\% de los sujetos con un destacado desempeño obtuvieron una evaluación sobresaliente en inteligencia emocional; apenas el 10\% de los individuos con resultados bajos obtienen una valoración mayor, por lo tanto la inteligencia emocional permite obtener un mayor puntaje.

El estudiantado del segundo año de bachillerato en ciencias de la Unidad Educativa Aida León de Rodríguez Lara tiene dificultades con el manejo de las emociones, como lo son: estrés, menor asertividad, reprimir emociones, conclusiones apresuradas, etc. Además, genera un retroceso en las distintas etapas del pensamiento crítico y los maestros lo relacionan como algo habitual y definitivo entre los estudiantes. Según Jayasankara et al (2018) afirman que el estrés es habitual en los estudiantes universitarios por altas expectativas tanto externas e internas.

La poca asertividad de los estudiantes según Thenmozhi (2019) no incitan esa destreza social que fomenta la tranquilidad, cuando los estudiantes no expresan sus emociones. Según Seli et al (2016) enuncian que las emociones impulsan la concentración de los estudiantes y desarrolla el proceso de aprendizaje, para Hasanpour (2018); las destrezas del pensamiento crítico y la inteligencia emocional es una de las metas de la educación, para Tevdovska (2016) en el entorno pedagógico incentivar la inteligencia emocional se ha convertido en un labor inevitable, la valoración de esta destreza es primordial para el desarrollo emocional y social de los individuos. 
El objetivo general es plantear un programa de inteligencia emocional para incrementar y mejorar el pensamiento crítico de los estudiantes de segundo año de bachillerato en ciencias de la Unidad Educativa Aida León de Rodríguez Lara, Guayaquil 2021. Del mismo modo, los objetivos específicos se proponen los siguientes: 1.- Examinar las peculiaridades de la inteligencia emocional de los estudiantes de segundo año de bachillerato en ciencias; 2.- Determinar las características del pensamiento crítico que presentan los estudiantes de segundo año de bachillerato en ciencias; 3.- Elaborar un programa de inteligencia emocional para incrementar y mejorar el pensamiento crítico de los estudiantes de segundo año de bachillerato en ciencias; 4.- Validar el programa de inteligencia emocional para incrementar y mejorar el pensamiento crítico de los estudiantes de segundo año de bachillerato en ciencias.

De acuerdo con Gadamer (1977) en su estudio acerca del pensamiento crítico indagó sobre el enfoque crítico del contexto desde una visión literal y clara. Desde el punto de vista de Sternberg (1986) las teorías educativas del pensamiento crítico parten desde la observación y la practica durante el desarrollo de la clase.

\section{ESTRATEGIAS METODOLÓGICAS O MATERIALES Y MÉTODOS}

La singularidad de esta investigación se debe a que fue aplicada y de tipo descriptivo, el Consejo Nacional de Ciencia, Tecnología e Innovación Tecnológica ([CONCYTEC], 2018) expresa que la investigación aplicada sirve para establecer, usando el conocimiento; las herramientas que proporcionen corregir un problema en específico. Desde la posición de Díaz et al (2016) la investigación descriptiva es un tipo de investigación donde se acude cuando se menciona las características de una población, se recolectan datos para dar respuestas a diversas preguntas referentes a un grupo concreto. El enfoque de la presente investigación fue de tipo cuantitativo, siendo Disman y Barliana (2017) afirma que la diferencia entre la investigación cualitativa, de la cuantitativa es que se hace cargo de datos numéricos o tiene algún tipo de relación con el cambio de cantidades.

El diseño de esta investigación fue no experimental de corte transversal; según Mohajan (2021) declara que una investigación no experimental escasea de manipulación de la variable independiente, logrando que el investigador elabore una comprobación de las variables tal cual como estas acontecen, la motivación, es el ánimo que interviene en el 
individuo para que ejecute gestiones e insista y así conseguir los objetivos deseados, sus indicadores son la iniciativa, compromiso y optimismo.

Asiamah et al (2017) consideran que la población es un estado de individuos con cosas en común y estos son de interés, puede implicar credibilidad de la investigación en relación a la noción, definición y elección del investigador; para la presente investigación se seleccionó a los estudiantes matriculados en el segundo año de bachillerato en ciencias de la Unidad Educativa Aida León de Rodríguez Lara, para Garg (2016) el criterio de inclusión se da cuando se determina la población objetiva, el investigador debe valorar si es viable examinar a cada uno delos individuos para conseguir un resultado; para este estudio fueron seleccionados 60 estudiantes del curso A y B de la institución y nivel educativo establecidos. Según Moreno Guillen (2017) considera una muestra censal a una población pequeña y finita y por lo tanto se tomará cada uno de los individuos de dicha población.

Para la ejecución de esta investigación, se recurrió a un muestreo probabilístico aleatorio simple West (2016) afirma que este muestreo es donde uno o todo el conjunto de integrantes de una población tienen la misma probabilidad de ser admitidos en la muestra y donde las posibles muestras tienen una posibilidad de ser elegidas.

Para las variables de inteligencia emocional y pensamiento crítico se empleó la encuesta que según Stevanov y otros (2015) menciona que es la practica más utilizada en las ciencias sociales y tiene como objetivo hacer suposiciones de una población bajo estudio. Para análisis de los datos recolectados en la encuesta, se planteó del programa estadístico IBM SPSS Statistics 26, es un paquete informático que realiza diversos estudios estadísticos que se deben emplear en la elaboración de una investigación y los resultados se consiguen en un corto tiempo. Para la validez de criterio se observó las dimensiones de cada variable de la investigación, se precisó de un número de indicadores con la intención de dar cumplimiento a los objetivos; la variable inteligencia emocional requirió de 3 dimensiones y 9 indicadores; la variable pensamiento crítico abarcó 4 dimensiones y 12 indicadores.

Se manejó el Alpha de Cronbach que para Namdeo y Rout (2016) refiere a la prudencia en que cada uno de los elementos de un instrumento calcula un mismo concepto y, por ende, estos son asociados entre sí. Esta medida de consistencia interna debería ser determinada primero, para emplear la prueba en la investigación para responder la validez 
del instrumento. El Alpha de Cronbach para la variable independiente fue de 0,902 y para la variable dependiente 0,896 concluyéndose que los instrumentos utilizados son fiables. Los datos obtenidos en la encuesta de los estudiantes fueron clasificados dependiendo de las dimensiones, después se tabuló los datos utilizando una hoja de Excel para luego comparar en el programa SPSS 26, se establecieron tablas y figuras estadísticas; luego se usó la prueba Kolmogorov - Smirnov para observar la repartición de los datos, después se efectuó la prueba de validación de hipótesis por medio de Chi Cuadrado y lograr realizar suposiciones acerca de la investigación.

\section{RESULTADOS Y DISCUSIÓN}

Para efectuar una crítica de los resultados derivados delas encuestas se realizó la descripción estadística mediante la representación de los datos en tablas y graficas de las variables inteligencia emocional y pensamiento crítico según la valoración de los estudiantes del segundo de bachillerato especialización ciencias, Guayaquil 2021; con sus respectivas dimensiones.

\section{Tabla 1}

Nivel de las variables inteligencia emocional y pensamiento crítico.

\begin{tabular}{lcccc}
\hline \multirow{2}{*}{ Categorías } & \multicolumn{2}{c}{ Inteligencia emocional } & \multicolumn{2}{c}{ Pensamiento crítico } \\
\cline { 2 - 5 } & $\mathbf{N}^{\circ}$ & $\mathbf{\%}$ & $\mathbf{N}^{\circ}$ & $\%$ \\
\hline Bajo & 2 & $3 \%$ & 1 & $2 \%$ \\
Medio & 30 & $50 \%$ & 33 & $55 \%$ \\
Alto & 28 & $47 \%$ & 26 & $43 \%$ \\
\hline Total & $\mathbf{6 0}$ & $\mathbf{6 0}$ & $\mathbf{6 0}$ & $\mathbf{1 0 0 \%}$ \\
\hline
\end{tabular}

Nota: Instrumento de investigación. Elaborado por: Johanna del Carmen Romero Jiménez.

Los niveles de inteligencia emocional se dividieron en tres niveles, donde el $50 \%$ tiene un nivel medio de respuesta; el $47 \%$ obtuvo un nivel alto, y un 3\% de los estudiantes está en un nivel bajo de inteligencia emocional; las dimensiones para medir la variable son: la compostura, la motivación y las habilidades sociales. Los niveles de pensamiento crítico, se clasifican en tres niveles, donde el $55 \%$ de los estudiantes tienen un nivel medio de respuesta y el $43 \%$ alcanzó un nivel alto, y otro $2 \%$ de los estudiantes tienen un nivel bajo de pensamiento crítico, las dimensiones de las variables de medición fueron la evaluación, inferenciación, mente abierta y comunicación eficaz.

Tabla 2. Clasificación de frecuencia de la dimensión compostura. 
Nota: Instrumento de investigación. Elaborado por: Johanna del Carmen Romero

\begin{tabular}{lcc} 
& \multirow{2}{*}{ Categorías } & \multicolumn{2}{c}{ Estudiantes } \\
\cline { 2 - 3 } & $\mathbf{N}^{\circ}$ & $\mathbf{\%}$ \\
\hline Bajo & 2 & $3 \%$ \\
Medio & 31 & $52 \%$ \\
Alto & 27 & $45 \%$ \\
\hline Total & $\mathbf{6 0}$ & $\mathbf{1 0 0 \%}$ \\
\hline
\end{tabular}

Jiménez.

La tabla 2 indica que los estudiantes se encuentran en un nivel bajo de 3\%, medio en un $52 \%$; se debe a que, aunque parezcan relajados no tienen autocontrol cuando atraviesan episodios de estrés, tienden a irritarse si no están de acuerdo, además que un $45 \%$ tiene un nivel alto por la adaptación a cambios inesperados.

Tabla 3 Nivel de indicadores de la dimensión compostura.

\begin{tabular}{llcrrrrrr}
\hline \multirow{2}{*}{ Indicadores } & \multicolumn{2}{c}{ Bajo } & \multicolumn{2}{c}{ Medio } & \multicolumn{2}{c}{ Alto } & \multicolumn{2}{c}{ Total } \\
\cline { 2 - 10 } & $\mathbf{N}^{\circ}$ & $\mathbf{\%}$ & $\mathbf{N}^{\circ}$ & $\mathbf{\%}$ & $\mathbf{N}^{\circ}$ & $\mathbf{\%}$ & $\mathbf{N}^{\circ}$ & $\%$ \\
\hline Autocontrol & 3 & $5 \%$ & 42 & $70 \%$ & 15 & $25 \%$ & 60 & $100 \%$ \\
Adaptabilidad & 2 & $3 \%$ & 38 & $64 \%$ & 20 & $33 \%$ & 60 & $100 \%$ \\
Innovación & 1 & $2 \%$ & 40 & $67 \%$ & 19 & $31 \%$ & 60 & $100 \%$ \\
\hline
\end{tabular}

Nota: Instrumento de investigación. Elaborado por: Johanna del Carmen Romero Jiménez.

El indicador autocontrol, demostró los siguientes porcentajes 5\% bajo, 705 medio y $25 \%$ alto. De igual forma la adaptabilidad mostró porcentajes en un nivel bajo en un 3\%, medio en un $64 \%$ y alto en un 33\%. Por último, el indicador innovación el cual los estudiantes la valoraron con un nivel bajo en un $2 \%$, medio $67 \%$ y alto $31 \%$.

Tabla 4. Clasificación de frecuencia de la dimensión motivación.

Nota: Instrumento de investigación. Elaborado por: Johanna del Carmen Romero

\begin{tabular}{lcc} 
& \multirow{2}{*}{ Categorías } & \multicolumn{2}{c}{ Estudiantes } \\
\cline { 2 - 3 } & $\mathbf{N}^{\circ}$ & $\%$ \\
\hline Bajo & 1 & $2 \%$ \\
Medio & 34 & $57 \%$ \\
Alto & 25 & $41 \%$ \\
\hline Total & 60 & $100 \%$ \\
\hline
\end{tabular}

Jiménez.

La tabla 4 alude que los estudiantes se encuentran en un nivel medio de motivación con un $57 \%$, debido a que no tienen seguridad al momento de expresar soluciones a los 
problemas, un $41 \%$ se ubicó en un nivel alto, los estudiantes manifiestan sentirse feliz en el ambiente que se desenvuelven; por último, un $2 \%$ en un nivel bajo.

\section{Tabla 5}

Nivel de indicadores de la dimensión motivación.

\begin{tabular}{lcccccccc}
\hline \multirow{2}{*}{ Indicadores } & \multicolumn{2}{c}{ Bajo } & \multicolumn{2}{c}{ Medio } & \multicolumn{2}{c}{ Alto } & \multicolumn{2}{c}{ Total } \\
\cline { 2 - 9 } & $\mathbf{N}^{\circ}$ & $\%$ & $\mathbf{N}^{\circ}$ & $\%$ & $\mathbf{N}^{\circ}$ & $\%$ & $\mathbf{N}^{\circ}$ & $\%$ \\
\hline Iniciativa & 4 & $7 \%$ & 38 & $63 \%$ & 18 & $30 \%$ & 60 & $100 \%$ \\
Compromiso & 4 & $7 \%$ & 36 & $60 \%$ & 20 & $33 \%$ & 60 & $100 \%$ \\
Optimismo & 2 & $3 \%$ & 36 & $60 \%$ & 22 & $37 \%$ & 60 & $100 \%$ \\
\hline
\end{tabular}

Nota: Instrumento de investigación. Elaborado por: Johanna del Carmen Romero Jiménez.

El indicador iniciativo, presentó porcentajes que se descompusieron en un 7\% bajo, $63 \%$ medio y $30 \%$ alto; señala que los estudiantes toman acciones que les permitan emprender alguna cosa. El indicador compromiso, se distribuyó en un $7 \%$ bajo, $60 \%$ medio y $37 \%$ alto; constituye a la conducta positiva del estudiante frente a contextos negativos.

\section{Tabla 6}

Clasificación de frecuencia de la dimensión habilidades sociales.

\begin{tabular}{lcc} 
& \multirow{2}{*}{ Categorías } & \multicolumn{2}{c}{ Estudiantes } \\
\cline { 2 - 3 } & $\mathbf{N}^{\circ}$ & $\%$ \\
\hline Bajo & 2 & $3 \%$ \\
Medio & 30 & $50 \%$ \\
Alto & 28 & $47 \%$ \\
\hline Total & 60 & $100 \%$ \\
\hline
\end{tabular}

Nota: Instrumento de investigación. Elaborado por: Johanna del Carmen Romero Jiménez.

La tabla 6 muestra que los estudiantes concordaron en un nivel medio de habilidades sociales con un $50 \%$ ya que sienten que no poseen destrezas sociales para conseguir lo que se proponen, un $47 \%$ se situó en un nivel alto, los estudiantes suelen evaluar posibles propuestas de solución a los problemas; y un 3\% en un nivel bajo.

\section{Tabla 7}

Nivel de indicadores de la dimensión habilidades sociales.

\begin{tabular}{ccccc}
\hline Indicadores & Bajo & Medio & Alto & Total \\
\hline
\end{tabular}




\begin{tabular}{lcccccccc}
\hline & $\mathbf{N}^{\circ}$ & $\boldsymbol{\%}$ & $\mathbf{N}^{\circ}$ & $\boldsymbol{\%}$ & $\mathbf{N}^{\circ}$ & $\boldsymbol{\%}$ & $\mathbf{N}^{\circ}$ & $\boldsymbol{\%}$ \\
\hline Influencia & 17 & $28 \%$ & 35 & $58 \%$ & 8 & $14 \%$ & 60 & $100 \%$ \\
Liderazgo & 3 & $5 \%$ & 35 & $58 \%$ & 22 & $37 \%$ & 60 & $100 \%$ \\
Gestión de conflictos & 2 & $3 \%$ & 36 & $60 \%$ & 22 & $37 \%$ & 60 & $100 \%$ \\
\hline
\end{tabular}

Nota: Instrumento de investigación. Elaborado por: Johanna del Carmen Romero Jiménez.

Los indicadores de la dimensión de habilidades sociales, indica que los estudiantes no suelen encontrar la manera que las cosas salgan como desean y ocasiona frustración generando que se rindan y no consigan sus objetivos planteados. Simultáneamente, el hecho que manifestaron es que los compañeros de clase $\mathrm{u}$ otras personas no los escuchan, ni tampoco los resguardan, esto incrementa los problemas de comunicación social. Finalmente, no presentan facilidad para describir claramente los problemas lo cual no les permitirá tomar decisiones concretas.

\section{Tabla 8}

Nivel de indicadores de la dimensión evaluación.

\begin{tabular}{lcccccccc}
\hline \multirow{2}{*}{ Indicadores } & \multicolumn{2}{c}{ Bajo } & \multicolumn{2}{c}{ Medio } & \multicolumn{2}{c}{ Alto } & \multicolumn{2}{c}{ Total } \\
\cline { 2 - 9 } & $\mathbf{N}^{\circ}$ & $\mathbf{\%}$ & $\mathbf{N}^{\circ}$ & $\%$ & $\mathbf{N}^{\circ}$ & $\%$ & $\mathbf{N}^{\circ}$ & $\%$ \\
\hline Comparación & 1 & $2 \%$ & 31 & $52 \%$ & 28 & $48 \%$ & 60 & $100 \%$ \\
Diferenciación & 1 & $2 \%$ & 32 & $53 \%$ & 27 & $45 \%$ & 60 & $100 \%$ \\
Cuestionamiento & 3 & $5 \%$ & 25 & $42 \%$ & 32 & $53 \%$ & 60 & $100 \%$ \\
\hline
\end{tabular}

Nota: Instrumento de investigación. Elaborado por: Johanna del Carmen Romero Jiménez.

El indicador comparación, reveló valores que se distribuyeron en un 2\% bajo, $52 \%$ medio y $48 \%$ alto; este indicador señala la capacidad del estudiante en razonar detalladamente cada una de las opciones antes de tomar una postura. El indicador diferenciación, se distribuyó en un $2 \%$ bajo, $53 \%$ medio y $45 \%$ alto; este indicador indica a la habilidad que tienen los estudiantes en reconocer características distintivas en un contexto. El indicador cuestionamiento, se divido en un nivel bajo en un 5\%, medio en un $42 \%$ y un $53 \%$ alto, este indicador representa las acciones que los estudiantes toman cuando no entienden un tema en singular.

\section{Tabla 9}

Clasificación de frecuencia de la dimensión inferenciación.

\section{Categorías}

\section{Estudiantes}




\begin{tabular}{lcc}
\cline { 2 - 3 } & $\mathbf{N}^{\circ}$ & $\%$ \\
\hline Bajo & 1 & $2 \%$ \\
Medio & 32 & $53 \%$ \\
Alto & 27 & $45 \%$ \\
\hline Total & 60 & $100 \%$ \\
\hline
\end{tabular}

Nota: Instrumento de investigación. Elaborado por: Johanna del Carmen Romero Jiménez.

De acuerdo con la tabla 9, manifiesta que los estudiantes se encuentran en un nivel medio de inferenciación con un $55 \%$ ya que sienten que no tienen la capacidad de percibir cada uno de los escenarios posibles al tomar una decisión, un $43 \%$ se situó en nivel alto, ya que concluye con coherencia algún tema observado; y un $2 \%$ en un nivel bajo.

\section{Tabla 10}

Nivel de indicadores de la dimensión inferenciación.

\begin{tabular}{lcccccccc}
\hline \multirow{2}{*}{ Indicadores } & \multicolumn{2}{c}{ Bajo } & \multicolumn{2}{c}{ Medio } & \multicolumn{2}{c}{ Alto } & \multicolumn{2}{c}{ Total } \\
\cline { 2 - 9 } & $\mathbf{N}^{\circ}$ & $\mathbf{\%}$ & $\mathbf{N}^{\circ}$ & $\%$ & $\mathbf{N}^{\circ}$ & $\%$ & $\mathbf{N}^{\circ}$ & $\%$ \\
\hline Reconocimiento & 2 & $3 \%$ & 32 & $54 \%$ & 26 & $43 \%$ & 60 & $100 \%$ \\
Presunción & 2 & $3 \%$ & 36 & $60 \%$ & 22 & $37 \%$ & 60 & $100 \%$ \\
Conclusión & 1 & $2 \%$ & 17 & $28 \%$ & 42 & $70 \%$ & 60 & $100 \%$ \\
\hline
\end{tabular}

Nota: Instrumento de investigación. Elaborado por: Johanna del Carmen Romero Jiménez.

En relación a todos los indicadores que forman la dimensión inferenciación se comprobó que los estudiantes cuando no están de acuerdo con el contenido de algún texto no estipulan con sustentos la causa del rechazo. Del mismo modo, no distinguen las probables implicaciones a favor o en contra en cuanto a la solución de un problema. Para finalizar, sus participaciones dentro de clase no suelen concluir correctamente.

\section{Tabla 11}

Clasificación de frecuencia de la dimensión mente abierta. 
Nota: Instrumento de investigación. Elaborado por: Johanna del Carmen Romero Jiménez.

\begin{tabular}{lcc} 
& Categorías & \multicolumn{2}{c}{ Estudiantes } \\
\cline { 2 - 3 } & $\mathbf{N}^{\circ}$ & $\%$ \\
\hline Bajo & 2 & $2 \%$ \\
Medio & 34 & $57 \%$ \\
Alto & 25 & $42 \%$ \\
\hline Total & 60 & $100 \%$ \\
\hline
\end{tabular}

Con respecto a la tabla 11, se comprueba que la dimensión establecida denominada mente abierta en los estudiantes se encuentran en un nivel medio con un $53 \%$ debido a que no se sienten oportunos en cambiar su rutina diaria, un $43 \%$ se situó en un nivel alto, porque no se obstaculizan en los problemas que se les presentan; y un 3\% en un nivel bajo.

\section{Tabla 12}

Test Kolmogorov - Smirnov de las variables inteligencia emocional y pensamiento crítico.

\begin{tabular}{llll}
\hline \multirow{2}{*}{ Tests of Normality } & \multicolumn{3}{c}{ Kolmogorov-Smirnova } \\
\cline { 2 - 4 } & Statistic & N & Sig. \\
\hline Inteligencia emocional & 0,226 & 60 & 0,000 \\
Pensamiento crítico & 0,157 & 60 & 0,001 \\
\hline
\end{tabular}

Nota. Reporte SPSS. Elaborado por: Johanna del Carmen Romero Jiménez.

La tabla 12, establece que los datos presentan una repartición no normal para las variables inteligencia emocional y pensamiento crítico, por su grado de significancia es menor a 0.05; por lo tanto, para la ejecución de comprobación de la hipótesis de investigación se empleará el estadístico Chi - Cuadrado X2. La hipótesis de investigación fue la siguiente: Hi: La elaboración de un programa de inteligencia emocional mejorará e incrementará el pensamiento crítico de los estudiantes de segundo año de bachillerato en ciencias de la Unidad Educativa Aida León de Rodríguez Lara, Guayaquil 2021.

\section{Tabla 13}

Test de Chi Cuadrado.

\begin{tabular}{lcc}
\hline Test no paramétrico & Inteligencia emocional & Pensamiento crítico \\
\hline Chi-Square & 11,067 & 14,533 \\
\hline
\end{tabular}




\begin{tabular}{lc|c}
\hline df & 18 & 15 \\
\hline Asymp. Sig. & 0,000 & 0,015 \\
\hline
\end{tabular}

Nota. Reporte SPSS. Elaborado por: Johanna del Carmen Romero Jiménez.

El test Chi- Cuadrado lanzó un valor de significancia menor 0,05 para las dos variables; por tanto, se impugna la hipótesis nula y se admite la hipótesis de investigación, señalando que la elaboración de un programa de inteligencia emocional incrementará y mejorará el pensamiento crítico de los estudiantes de segundo año de bachillerato en ciencias de la Unidad Educativa Aida León de Rodríguez Lara, Guayaquil, 2021.

\section{CONCLUSIÓN O CONSIDERACIONES FINALES}

1) Un programa de inteligencia emocional ayudará a los estudiantes en concientizar sobre la importancia de efectuar la motivación, dominio de los impulsos, sobrellevar emociones y lograr una vinculación con quienes lo rodean en beneficio de su desenvolvimiento dentro y fuera de la institución educativa, su futuro, vida profesional y logrando posibilitar el pensamiento crítico como una medida de apoyo.

2) La particularidad que presentó la inteligencia emocional en los estudiantes de segundo año de bachillerato en ciencias es que no logran detenerse cuando exhiben sus pensamientos, invadiendo las consecuencias de sus actos suscitando problemas en la interrelación entre compañeros, no logran aportar ideas creativas ante situaciones de conflicto y para finalizar, no saben influir sobre las personas para que puedan liberarse de cualquier situación consiguiendo que las cosas funcionen.

3) Las características que mostro el pensamiento crítico de los estudiantes de segundo año de bachillerato en ciencias son que no logran distinguir información relevante, no prevén el contenido de un texto cuando no contiene la información completa, y para concluir los estudiantes no pueden comunicar sus ideas de manera asertiva y poseen poca motivación para lograr transmitir sus pensamientos o postura.

4) Mediante la verificación de los datos se logró observar un comportamiento normal, por lo tanto, se manejó como estadística para la elaboración de la prueba de hipótesis en el test de Chi Cuadrado donde el resultado para la inteligencia emocional lanza un grado de significancia del 0,000 y para el pensamiento crítico 0,015; ratificando que un programa de inteligencia emocional incrementará mejorará el pensamiento crítico de los estudiantes de segundo año de bachillerato en ciencias. 
5) En la elaboración de la propuesta se consideró las divisiones derivadas de la encuesta donde evidencia la capacidad media de inteligencia emocional con la que se desenvuelven los estudiantes de segundo año de bachillerato en ciencias, siendo indudable la necesidad de instaurar un programa de inteligencia emocional.

6) El programa de inteligencia emocional fue consolidado mediante el criterio de expertos quienes construyeron la disposición del trabajo realizado.

\section{LISTA DE REFERENCIAS}

[CONCYTEC]. (2018). Consejo Nacional de Ciencia, Tecnología e Innovación Tecnológica . Reglamento de Calificación, Clasificación y Registro de los Investigadores del Sistema Nacional de Ciencia, Tecnología e Innovación Tecnológica (CONCYTEC). Obtenido de https://portal.concytec.gob.pe/images/renacyt/reglamento_renacyt_version_final. pdf

[UNESCO]. (2017). Organización de las Naciones Unidas para la Educación, la Ciencia y la Cultura Obtenido de Unesco.org: http://www.unesco.org/new/fileadmin/MULTIMEDIA/FIELD/Santiago/pdf/Gui a_3_web_educacion_emergencias.pdf

Akbarilakeh, M., Naderi, A., \& Azizollah, A. (2018). Critical Thinking and Emotional Intelligence Skills and Relationship with Students' Academic Achievement. La Prensa Médica Argentina, $\quad$ 104(2), 1-5. doi:http://dx.doi.org/10.4172/lpma.1000280

Asiamah, N., Mensah, H., \& Oteng-Abayie, E. (2017). General, Target, and Accessible Population: Demystifying the Concepts for Effective Sampling. TQR, 22(6), 1607 - 1622. doi:https://doi.org/10.46743/2160-3715/2017.2674

Díaz-Narváez, V., \& Calzadilla Núñez, A. (2016). Artículos Científicos, Tipos de Investigación y Productividad Científica en las Ciencias de la Salud. Rev Cienc Salud, 115 - 121.

Disman, M., \& Barliana, S. (2017). The Use of Quantitative Research Method and Statistical Data Analysis in Dissertation: An Evaluation Study. International Journal of Education, 10(1). doi:http://dx.doi.org/10.17509/ije.v10i1.5566

Fernández-Martínez, A., \& Montero-García, I. (2015). Aportes para la Educación de la Inteligencia Emocional desde la Educación Infantil . Revista Latinoamericana de 
$\begin{array}{llllll}\text { Ciencias } & \text { Sociales } & , & 14(1), & 53 & -\end{array}$ doi:https://doi.org/http://dx.doi.org/10.11600/1692715x.1412120415

Gadamer, H. (1977). Verdad y Método: Fundamentos de una Hermenéutica Filosófica (1 era ed.). Editorial Sígueme. Obtenido de https://www.academia.edu/9082328/Verdad_y_M\%C3\%A9todo_I_Hans_Georg _Gadamer_

Garg, R. (2016). Methodology for research. Indian Journal Anaesthesia, 60(9), 640 - 645. doi:http://dx.doi.org/10.4103/0019-5049.190619

Hasanpour, M., Maryam, B., \& Ghaedi, F. (2018). The relationship between emotional intelligence and critical thinking skills in Iranian nursing students. Medical Journal of The Islamic Republic of Iran, 32(40), 32- 40. doi:http://dx.doi.org/10.14196/mjiri.32.40

Jayasankara Reddy, K., Rajan Menon, K., \& Thattil, A. (2018). Academic Stress and its Sources Among University Students. Biomedical and Pharmacology Journal, 11(1), 531-537. doi:https://dx.doi.org/10.13005/bpj/1404

Miners, C., \& Hideg, I. (2015). Emotional Intelligence and Competencies. Elseiver, 7(2), 445 - 451. doi:http://dx.doi.org/10.1016/B978-0-08-097086-8.25053-8

Mohajan, K. (2021). Journal of Economic Development Environment, 9(4), 50-79. doi:http://dx.doi.org/10.26458/jedep.v9i4.679

Moreno Guillen, R. (2017). Líneas Estratégicas de Comunicación en el Desarrollo de Habilidades Gerenciales y Humanas. Revista Scientific, 2(1), 376 - 392. doi:https://doi.org/10.29394/scientific.issn.2542-2987.2017.0.0.20.376-393

Namdeo, S., \& Rout, S. (2016). Calculating and Interpreting Cronbach's Alpha using Rosenberg Assessment Scale on Paediatrician's Attitude and Perception on Self Esteem. International Journal of Community Medicine and Public Health, 3(6), 1371 - 1374. doi:http://dx.doi.org/10.18203/2394-6040.ijcmph20161448

Seli, P., Wammes, J., Risko, E., \& Smilek, D. (2016). On the relation between motivation and retention in educational contexts: The role of intentional and unintentional mind wandering. Psychonomic Bulletin \& Review, 23(4), 1280-1287. doi:https://doi.org/10.3758/s13423-015-0979-0 
Sternberg, R. (1986). Critical thinking: Its nature, measurement, and improvement . National Institute of Education, 1(1), 1 - $36 . \quad$ Obtenido de https://files.eric.ed.gov/fulltext/ED272882.pdf

Stevanov, M., Dobšinská, Z., \& Ostoić, S. (2015). -Survey Research in the Forest Science Journals Insights from Insights from South. East European Forestry, 6(2), 237 247.

Tevdovska, E. (2016). The Impact of Emotional Intelligence in the Context of Language Learning and Teaching. SEEU Review, 12(1). doi:http://dx.doi.org/10.1515/seeur-2017-0009

Thenmozhi, P. (2019). Relationship between the Level of Assertiveness and Academic Performance among Arts and Science College students. Asian Journal of Nursing Education and Research, 9(3), 2349-2996. doi:http://dx.doi.org/10.5958/23492996.2019.00063.6

Trotta, J. (10 de Diciembre de 2018). Linkedin. Obtenido de https://www.linkedin.com/pulse/emotional-intelligence-what-do-numbers-meanjoanne-

trotta\#: :text=90\%25\%20of\%20top $\% 20$ performers $\% 20$ score, will\%20become $\%$ 20a\%20top\%20performer.

West, P. (2016). Simple random sampling of individual items in the absence of a sampling frame that lists the individuals. West New Zealand Journal of Forestry Science, 46(15), 1 - 7 . doi:https://doi.org/10.1186/s40490-016-0071-1 